\title{
Rheumatic disease management in the Campania region of Italy during the COVID-19 pandemic
}

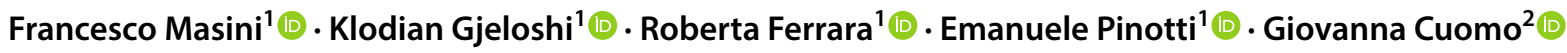

Received: 2 June 2020 / Accepted: 7 July 2020 / Published online: 14 July 2020

(c) Springer-Verlag GmbH Germany, part of Springer Nature 2020

Keywords COVID-19 $\cdot$ Management $\cdot$ Rheumatic diseases $\cdot$ Pandemic $\cdot$ Rheumatology

\section{Dear Editor,}

Severe acute respiratory syndrome coronavirus 2 (SARSCoV-2), also called COVID-19 disease, was firstly reported in December 2019 in China and soon declared a pandemic by the World Health Organization (WHO) on March 11, 2020. In Italy, on June 14, 2020, we report 236,989 infected patients and 34,345 deaths. Although Campania Region is among the less affected regions, 4609 SARS-Cov- 2 cases have occurred. 2019-nCoV, a single-strand RNA virus responsible for COVID-19, spreads by human-to-human transmission. Most common transmission vehicles are respiratory droplets, aerosols and conjunctiva, but the virus can also persist on surfaces. COVID-19 may be asymptomatic till severe illness, i.e., severe pneumonia and death $[1,2]$.

Due to the rapid outbreak of COVID-19, concern has been growing about the management of rheumatic patients, particularly due to risk of infection and immunosuppressive treatments. Rheumatic patients taking immunomodulators

Giovanna Cuomo

giovanna.cuomo@unicampania.it

Francesco Masini

masini.fr@gmail.com

Klodian Gjeloshi

klodian87@gmail.com

Roberta Ferrara

robyferrara84@gmail.com

Emanuele Pinotti

emanuelepinotti@yahoo.it

1 Department of Advanced Medical and Surgical Sciences, "Luigi Vanvitelli” University of Campania, Piazza Luigi Miraglia 2, 80138 Naples, Italy

2 Department of Precision Medicine, "Luigi Vanvitelli" University of Campania, Via De Crecchio 7, 80138 Naples, Italy may be more susceptible to the infection, mainly in combination with biologic drugs [3]. Unlike other viruses (e.g., influenza), coronaviruses have not been shown to evolve to the more severe stages of the disease in immunosuppressed patients [4]. Usually, discontinuing therapeutic treatment is not recommended, since clinical flares of rheumatic disease develop with subsequent use of other immunosuppressants such as corticosteroids, equally unsafe in the case of COVID-19 occurrence [3]. Indeed, the risk of infection in rheumatic patients (i.e., rheumatoid arthritis) is also related to disease activity and a flare due to therapy interruption would confer an increased risk of infection. The European League Against Rheumatism (EULAR) and the Italian Society of Rheumatology (SIR) clinical guidance during COVID-19 pandemic recommended management of patients on long-term corticosteroid therapy by gradually tapering doses, and warning against abrupt cessation of corticosteroid therapy, even during active infection. Discontinuation of disease-modifying antirheumatic drugs, biologics, small molecules, and other immunosuppressive agents has been suggested just in case of overt infection [5]. SIR has also promoted a dedicated platform, "CONTROL-19", for the remote monitoring of COVID-19 impact on rheumatic diseases [6]. In our internal medicine outpatient, we have closely monitored patients with severe active disease either via phone-calls or e-mail, rescheduling the great majority of appointments and implementing restrictive measures at a social level [7]. We have selected patients that could not postpone their infusion, providing them with specific recommendations. In cases of extremely urgent need to initiate treatment, subcutaneous infusions were preferred over intravenous biologics, as they can be taken at home. An objective phone interview has been carried out before admission to assess for acute respiratory symptoms, and/or fever, and exclude contact with positive COVID-19 patients during the past 14 days. Infusions have been provided in a dedicated 
room, with access reserved only to one doctor and a nurse, equipped with individual protection devices to detect rapid IgM-IgG SARS-CoV-2 antibody test. No accompanying person was allowed. A three-layer surgical mask, hand sanitizer and a disposable glove box was available at the entrance of the dedicated area. In the case of positive history, COVID-19 like symptoms or history of contact, both patient and attendant were isolated and treated as COVID-19 positive unless otherwise proven. Our protocol enforces to inform hospital administration authority [8-10].

Although more evidence is required to demonstrate the real impact of this strategy, telemedicine has demonstrated as a valid alternative to improve the quality of rheumatic patients' care during COVID-19 pandemic, thus reducing hospitalizations only to urgent admissions. We surprisingly report only one patient contacting us due to COVID-19 infection, and a limited number of urgent admissions during the lockdown period. Since the clinical examination and psychological implications derived from this period remain of fundamental importance, it would be useful to invest in new personalized patients' care strategies. Further studies would be worthied to establish appropriate and evidencebased strategies to be prepared to face with a likely second cycle of pandemic, expected for next autumn.

\section{Compliance with ethical standards}

Conflicts of interest "The authors have no conflict of interest to disclose."

\section{References}

1. Zhang J, Litvinova M, Wang W, Wang Y, Deng X, Chen X, Li M, Zheng W, Yi L, Chen X, Wu Q, Liang Y, Wang X, Yang J, Sun K,
Longini IM Jr, Halloran ME, Wu P, Cowling BJ, Merler S, Viboud C, Vespignani A, Ajelli M, Yu H (2020) Evolvingepidemiology and transmission dynamics of coronavirus disease 2019 outside Hubei province, China: a descriptive and modelling study. Lancet Infect Dis. https://doi.org/10.1016/S1473-3099(20)30230-9

2. Qiu C, Deng Z, Xiao Q, Shu Y, Deng Y, Wang H, Liao X, Liu H, Zhou D, Zhao X, Zhou J, Wang J, Shi Z, Long D (2020) Transmission and clinical characteristics of coronavirus disease 2019 in 104 outside-Wuhan patients China. J Med Virol. https://doi. org/10.1002/jmv.25975

3. Ceribelli A, Motta F, De Santis M, Ansari AA, Ridgway WM, Gershwin ME, Selmi C (2020) Recommendations for coronavirus infection in rheumatic diseases treated with biologic therapy. J Autoimmun 109:102442. https://doi.org/10.1016/j. jaut.2020.102442

4. Pope JE (2020) What does the COVID-19 pandemic mean for rheumatology patients? Curr Treatm Opt Rheumatol 30:1-4. https ://doi.org/10.1007/s40674-020-00145-y

5. Jani M, Barton A, Hyrich K (2019) Prediction of infection risk in rheumatoidarthritis patients treated with biologics: are we any closer to riskstratification? Curr Opin Rheumatol 31(3):285-292. https://doi.org/10.1097/BOR.0000000000000598

6. EULAR (2020) EULAR guidance for patients during Covid-19 outbreak (2020). Publishing EULAR Web https://www.eular.org/ eular_guidance_for_patients_covid19_outbreak. (Accessed 19 Mar 2020)

7. SIR (2020) COVID-19 monitoring in patients with rheumatic and musculoskeletal diseases. Publishing SIR Web https://www.reuma tologia.it/cmsx.asp?IDPg=1107. (Accessed 15 Jun 2020)

8. Dumusc A, Dan D (2020) Rheumatology and COVID-19. Rev Med Suisse 16(2):831-834

9. Favalli EG, Ingegnoli F, Cimaz R, Caporali R (2020) What is the true incidence of COVID-19 in patients with rheumatic diseases? Ann Rheum Dis. https://doi.org/10.1136/annrheumdis-2020217615

10. Lewandowski LB, Hsieh E (2020) Global rheumatology in the time of COVID-19. Lancet Rheumatol 2(5):e254-e255. https:// doi.org/10.1016/S2665-9913(20)30091-6

Publisher's Note Springer Nature remains neutral with regard to jurisdictional claims in published maps and institutional affiliations. 